\title{
Fournier's gangrene
}

\author{
Phoebe S. Y. Cheung • Colin A. Graham
}

Received: 4 March 2009 / Accepted: 13 June 2009 / Published online: 4 August 2009

(C) Springer-Verlag London Ltd 2009

An 80-year-old diabetic man (type 2) with detrusor underactivity treated by long-term urethral catheterization attended the emergency department with a 4-day history of scrotal swelling and pain and an associated offensive discharge. Physical examination revealed a grossly swollen right hemi-scrotum with gangrene. Crepitus and erythema were noted over the discolored area, extending into the right groin. The diagnosis of Fournier's gangrene was made, and an intravenous cephalosporin and metronidazole were administered before proceeding to emergency partial

P. S. Y. Cheung $\cdot$ C. A. Graham $(\bowtie)$

Accident \& Emergency Medicine Academic Unit,

Chinese University of Hong Kong,

Hong Kong SAR, People's Republic of China

e-mail: cagraham@cuhk.edu.hk

P. S. Y. Cheung $\cdot$ C. A. Graham

Trauma and Emergency Centre, Prince of Wales Hospital,

Shatin, New Territories,

Hong Kong SAR, People's Republic of China scrotectomy. Operation revealed a $4 \times 6-\mathrm{cm}$ patch of gangrenous scrotal skin with inflammation of the right groin and suprapubic area. Pockets of purulent material extending from the scrotal wall to the inguinal canal were drained. The right testis was placed in the adductor canal. Morganella morganii and Peptostreptococcus spp. were cultured from the wound. He was treated with intravenous meropenem and subsequently improved. He was discharged home after 21 days (Fig. 1).

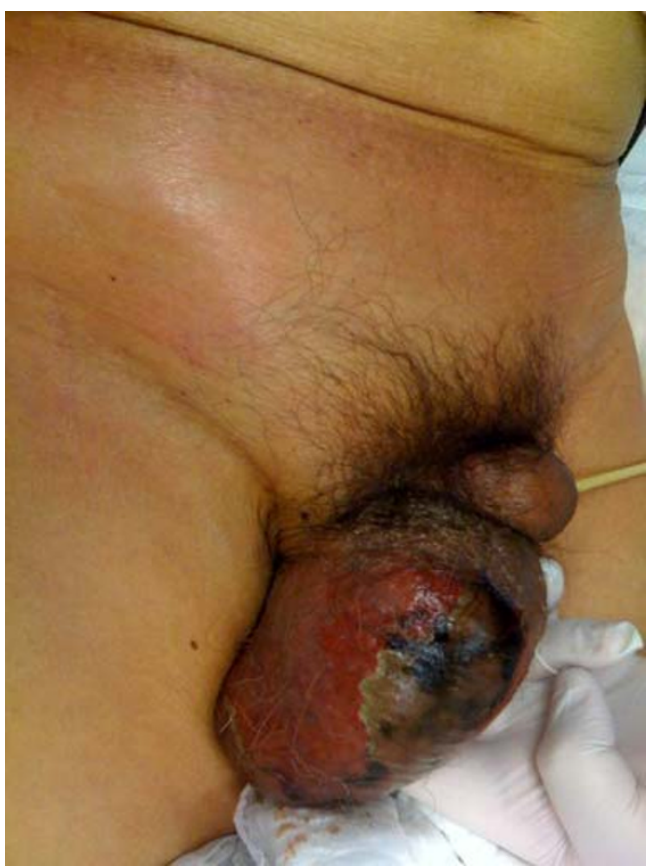

Fig. 1 Scrotum and groin in diabetic patient with long-term urethral catheter and Fournier's gangrene 